\title{
NEUROINNOVATIONS: \\ DRIVING CREATIVITY IN ORGANIZATIONS \\ THROUGH THE APPLICATIONS OF THE HUMAN NERVOUS SYSTEM AND THE BRAIN
}

\author{
Kwanhathai Tanmanee \\ Master of Science in International Management \\ Faculty of Business Administration \\ Hochschule Furtwangen University, Germany
}

\author{
Prof. Dr. Eva Kirner \& \\ Prof. Dr. Daniel Cerquera \\ Faculty of Business Administration \\ Hochschule Furtwangen University, Germany
}

In today's business world characterized by high level of uncertainty and change, the success and perhaps the survival of organizations rest on their ability to think and act creatively. Stimulating employee creativity, thus, inevitably becomes one of the top priorities for most organizations, no matter large or small. However, driving creativity is not an easy task, especially in an organizational context where creativity is influenced by variables operating at different levels. Therefore, what organizations really need are reliable, scientifically proven mechanisms and tools that would effectively boost the creativity of their employee. This is what the present study promises to deliver. To achieve this, the study analyzed and integrated current diverse and fragmented findings from organizational creativity research field, as well as insights from the field of neuroscience, so that a more complete understanding on how to drive creativity can be obtained. Understanding creative behaviors at their very neural substrates provide a great opportunity to draw more accurate conclusions. Therefore, the strategies this study ought to provide are not ones that are general, but brain-targeted ones. To test the stimuli identified from such analysis, experiments with 54 participants for individual variables, and 18 groups for group variables were carried out. In addition, two experts from IBM were being interviewed for their views on how IBM boosts creativity of their employees. Based on the experiments and interview results, four major recommendations were implicated. First, organizations should look for individuals who are open to experiences, are extraverted, and are not conscientious to work on the creative tasks. Second, organizations should encourage employees to individually work on their idea generation activity. Third, for group creative problem solving, change in membership should be encouraged as opposed to static membership. Last but not least, the cultures and climates inside the organizations should be ones that encourage transformational leadership and collaboration among employees. Also, a relaxed atmosphere does not necessary drive employees to be more creative. However, the flexibility, the autonomy provided to employees tends to do so.

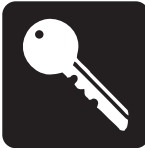

Creativity, Innovation, Creative Thinking, Organizational

Creativity, Neuroscience of Creativity, Organizational

Interventions 


\section{INTRODUCTION}

\section{I.1. BACKGROUND}

$\mathrm{T}$

he only thing constant in life is change" (Goodreads Inc., 2014). This statement, dated hundreds of years back have never been more suited than it is to the business world today. Characterized by an ever-expanding global nature, rapid changes in technology, and increasingly fierce competitions, such environment of high uncertainty and change presents challenges to businesses of both large and small (Florida, 2013). To keep up with the pace of such change, business therefore must remain in a continuous process of innovation, constantly developing the capacity to initiate or quickly implement new ideas. In fact, innovation and creativity have been proven critical to organizational growth and success, as have been exemplified in the cases where organization's rate of innovations are strongly associated to its profitability and the cases where the rate at which new products flow to the market significantly correlated to the financial performance of high technology firms (Geroski, Machin, \&Van Reenen, 1993; Eisenhardt \& Tabrizi, 1995).

As the prosperity and survival of today's businesses are increasingly depending on its ability to be creative, stimulating employee creativity thus become one of the major focuses of any organization. Specifically, such demands had driven organizations to initiate and implement various organizational practices, creating climate and culture that would best bring out the creative potentials of their employees. Do these investments really paid off though, is an interesting question. Recent studies suggest that they actually might not. The 2013 survey conducted by Accenture on more than 500 executives from across a wide range of sectors including Retail, Banking and Capital Markets, Electronics \& High Tech, and Consumer Goods revealed that more than half of these companies still struggle to innovate, specifically labeled their innovation process as "sluggish", despite the majority of them rated innovation as their top strategic priority and said that they strongly depend on innovation for their long-term success (Koetzier \& Alon, 2013).

Creativity is the product of the mind and the brain. In order to fully understand how to drive creative thinking, it would be wise to investigate what exactly happens inside the brains during creative process. Recent advances in the study of neuroscience may provide just the perfect tool to do so. Neuroscience, as generally defined, is the study of the development, structure and function of the nervous system, with a special focus on the brain and its role in behaviors and cognitive functions (BTNRC, 2013). Neuroscience complements research of organizational creativity as it looks deeper and from a different angle into the behaviors of individuals (Becker, Cropanzano \& Sanfey, 2011). By looking into the very neural substrates of such behaviors, it provides a great opportunity to determine what really drive individuals to be creative, what really drive their brains to be creative to be exact.

\section{I.2. RESEARCH AIM}

The main aim of the present research is to draw upon different levels of analysis for a more complete understanding on the phenomenon as complex as creativity. On one hand, the author aims to thoroughly investigate the field of organizational creativity, with the strong ambition to determine what have been suggested so far as factors contributing to creativity, and 
what still required further investigation. On the other hand, the author would like to gain a comprehensive understanding on the structures and functions of the human brains, in order to determine what attract them to be creative, and in contrast what prevent them to do so. Once knowledge of these two distinct fields have been obtained and combined, insights can be drawn and use as the basis to develop mechanisms and interventions organizations can use to enhance creative abilities of their employees. Through the back up of robust scientific findings, the author wishes to provide a helping hand to practitioners who currently seem to be isolated with the misconceptions in their heads. That is, on the high level, the author aims to reduce or even eliminate the gap between what science knows, and what business actually does.

\section{I.3. RESEARCH QUESTION}

By translating the aims into more operationalized focus, the key question that the research will address is:

What are the ideal organizational mechanisms or interventions organizations should implement, that would best target the brain, bringing out its creativity and ultimately fostering innovation in organizations?

\section{I.4. RESEARCH OBJECTIVES}

In order to address the research question stated above, the objectives are:

1. Organizational Creativity Field: Identify factors contributing to employees creativity in organizational contexts

2. Neuroscience Field: Examine the nature of the human brain, with the focus on how its structures and functions may impact creative abilities of individuals
3. Combine insights from organizational creativity and neuroscience. Identify organizational stimuli that would best bringing out the creativeness of the brains

4. Design and conduct measures to test the effectiveness of the stimuli identified

5. Based on the tested stimuli, proposed practices organizations should adopt in order to stimulate the creativity of their employees

\section{LITERATURE REVIEW}

In accordance to the research question of this study, there are two main areas where literature reviews are critical. First, is the review on "creativity in organizations". This means that literatures involving creativity in general, as opposed to creativity that are operationalized specifically in an organizational context, were excluded. Fundamentally, the review focused on determining what the current research identifies as the potential factors to enhance creativity in organizations, as well as those that are potential inhibitors. The second area of focus is "neuroscience of creativity". The review focused on identifying the nature of the human brain, and how it potentially benefits or diminish creativity.

\section{II.1. SUMMARY OF CREATIVITY LITERATURE REVIEW}

The published knowledge of creativity in organizational context is very diverse and fragmented. The detailed literature review on key relevant studies provided an integrated picture of what has been done so far in this field. In general, it can be concluded that there has already been done a great deal into understanding organizational creativity from social and organizational psychology perspectives. This, however, until now did not result in valid and reliable models and 
corresponding theories to effectively boost creativity within organizations. That is, despite their best efforts, researchers still try to answer the simple question of what facilitates and inhibit creativity in organizations. There are still ongoing debates among researchers on various variables. These include, on the individual level of analysis, which personalities enhance one's creativity, and whether activated moods boost creativity or not. At the team level which is relatively under researched, inconsistencies can be found on factors such as group diversity, group membership change, and the idea generation method. At the organizational level, variables such as leaderships style, competition, and pressure are at the central discussions and debates among researchers. These empirical evidences are not only lacking in number and consistency relative to their importance, the methodologies adopted by these researchers required further investigation as well. for instance, comparing results between different groups of people where their personal characteristics may impact the final outcomes, and some studies used only one gender or used both genders but with different number of people represented each gender. In addition, most researchers seem to have a bias for one given method over the others, that is, most of them seemed to use self-report for all measures of creativity and the organizational variables. Some studies also did not test directly the relationship between creativity and the variable of interest but instead using proxies for creativity such as organizational financial performance or team productivity. Furthermore, most of the researches available were conducted many years ago, which may resulted in findings not applicable to the present days. The summary of evaluation on each organizational variable literature is presented in table 1 below.

\section{Table 1. Summary of Organizational Creativity Literatures Evaluation}

\begin{tabular}{|l|c|c|c|c|}
\hline $\begin{array}{c}\text { Organizational Creativity } \\
\text { Variable }\end{array}$ & $\begin{array}{c}\text { Empirical Evidence: } \\
\text { Low/Medium/High }\end{array}$ & $\begin{array}{c}\text { Findings: } \\
\text { Level of Consistency } \\
\text { Low/Medium/High }\end{array}$ & $\begin{array}{c}\text { Methodology } \\
\text { Reliable \& Valid }\end{array}$ & $\begin{array}{c}\text { Test Direct } \\
\text { Relationship With } \\
\text { Creativity }\end{array}$ \\
\hline 1.Creative Personality & High & Medium & No & Yes \\
\hline 2.Knowledge & Medium & High & Yes & Yes \\
\hline 3.Motivations & High & High & Yes & Yes \\
\hline 4.Moods \& Emotions & Medium & Low & Yes & Yes \\
\hline 5.Group Diversity & Low & Low & No & No \\
\hline 6.Membership Change & Low & Medium & No & No \\
\hline 7.Psychological Safety & High & High & Yes & Yes \\
\hline 8.Brainstorming & Low & Medium & No & Yes \\
\hline 9.Leadership Style & Low & Medium & Yes & Yes \\
\hline 10.Competitive Environment & Low & Low & No & Yes \\
\hline 11. Stress \& Pressure & Medium & Low & Yes & Yes \\
\hline
\end{tabular}

For example, some studies did not control the background of their samples,
Therefore, what can be concluded is, many of the existing work lack support 
in experimental figures, or their tests produce results that are contradictory. Far more well-designed theoretical and empirical work, thus, is necessary to develop a thorough understanding on this multi-faceted construct of creativity.

\section{II.2. SUMMARY OF NEUROSCIENCE OF CREATIVITY LITERATURE REVIEW}

By conducting a critical review on the neuroscience literatures, the author found one common but very important insight: Brain's number one priority is survival. There are three properties of the brain that helps it achieve this task. First, brain as an emotional organ has a defensive threat response system to deals with fear and stress. Specifically such system helps one to fight harder or flee faster, thus increasing one's chance for survival. Although important to survival, the activation of such response may negatively impact creativity in many ways. For instance, when the brain devotes its resources to such activity, there is hardly any capacity left for the high energy consumption task like creative thinking. Also, the spread of neural networks becomes narrowed thus reduced the chance for remote associations to emerge. Second, brain as a predictive organ tries to increase its chance for survival by conserving energy as much as possible. This is done through mechanisms such as predictive coding and latent inhibitions which use knowledge and past experiences to carry out their processes. What this could mean to creativity is, creative thinking maybe constrained to what one already known, already experienced. Third, the social brain strives for relatedness, fairness, and high social status to increase its chance for survival. Therefore, anything that favors social interactions will enhance creativity as this activates the reward system and anything that diminish social interactions will decrease creativity as this activates the threat response. All of these conclusions, even though some are more supported by empirical evidences than others, together provide valuable insights to integrate with what the researchers from the organizational creativity already know.

\section{METHODOLOGY}

The in-depth analysis on organizational creativity literatures revealed a number of important factors that may enhance or hinder the creative abilities of individuals in an organizational setting. However, drawing definite conclusions from such work has proved to be difficult, as the empirical evidences are still very much lacking in numbers. Not only quantity, but quality- wise, most of these findings were often inconsistent, and some of the methodologies adopted were low in validity and reliability. Moreover, it was evident that a number of studies did not investigate the direct effects of variables in question on creativity, but rather on factors like the productivity of the teams or financial performances of organizations (see table 1 for the summary of evaluation).

As illustrated in table 1, most of the variables, with the exception of knowledge, motivations, and psychological safety required further investigations. To address the issues found in the previous studies, the present research adopted measures with improved validity and reliability. Insights from the review of neuroscience literatures were also incorporated into setting up the hypotheses of this study. 


\section{III.1. HYPOTHESES}

H1.1: Individuals who rely less on their past experiences and knowledge, tend to be ones that are more open to experience than individuals who rely more on their past experiences and knowledge

H1.2: Individuals who rely less on their past experiences and knowledge, tend to be ones that are more extraverted than individuals who rely more on their past experiences and knowledge

H1.3: Individuals who rely less on their past experiences and knowledge, tend to be ones that are less conscientious than individuals who rely more on their past experiences and knowledge

$\mathrm{H} 2$ : Individuals are likely to be more creative when they take a break, as opposed to when they are not

H3: Heterogeneous groups are likely to be more creative than homogenous groups

H4: When group experiences membership change, it is likely to be more creative than when it does not experience such a change

H5: Idea generation in the form of individual brainstorming is likely to lead to more creative solutions than idea generation in the form of group brainstorming

H6: Transformational leadership style is likely to enhance organizational creativity as opposed to transactional leadership style

H7: Competitive environment is likely to inhibit employees' creative thinking

H8: Organization with high-pressured work environment is likely to be less creative, as opposed to organization with a more relaxed atmosphere

\section{III.2. HYPOTHESES TESTING METHODS}

Two main data collection methods were utilized in this research: laboratory experiments and interviews.

\section{III.2.1. LABORATORY EXPERIMENTS}

Fifty-four individuals (27 men and 27 women) were randomly chosen to participate in the present study. All participants belonged to the working age group, with the average age of 27.6 years for women $(\mathrm{SD}=2.87)$, and 29.7 years for men $(\mathrm{SD}=4.23)$. The experiments were conducted between the end of January to early April, 2014.

At the beginning of the experiment, each participant was given a set of creative exercise sheets. A copy of such sheets can be found in Appendix A. The creative tasks used in this study were mainly adapted from the TTCT developed by psychologist Ellis Paul Torrance (Kim, 2006). This set of tests is arguably the most widely used test of individuals' capacity for creativity, both in the educational field and in the corporate world (Davis, 1997). TTCT involves simple tests of verbal and figural divergent thinking. For figural, participants are required to draw as many ideas as possible using the shape given, as well as giving titles for them. For verbal, they are to list out as many unusual uses as possible for the object given. Creative productions from the TTCT were scored on scales including fluency, flexibility, originality, resistance to premature closure, and abstractness of titles (Gifted Education, 2014). Fluency is the total number of interpretable, meaningful, and relevant ideas generated in response to the stimulus. Flexibility is the number of different categories of such relevant responses, and originality is the statistical 
rarity of the responses. Resistance to premature closure is the degree of psychological openness. Abstractness of titles is the degree a title moves beyond concrete labeling of the pictures drawn (Kim, 2006). In addition, they are scored on based on the creative strengths. These include emotional expressiveness (communication of feelings and emotions through titles and drawings), storytelling articulateness (put things in context, create an environment for the object so a story is told), movement/action (e.g. run, fly, float), expressiveness of title (transform visual information into emotions \& feelings expressed in words, go beyond simple description to express emotion and feeling), synthesis of incomplete figures (combination of two or more incomplete figures), unusual visualization (present ideas or objects in another view than the one we would ordinarily see), internal visualization (visualize beyond exteriors \& pay attention to the internal, dynamic workings of things, showing something inside or in cross- section), extending/breaking boundaries (extends the line, up, down, or out), humor (unusual combinations and surprises), richness of imagery (variety, vividness, liveliness, intensity, fresh visual images, memorable), and colorfulness of imagery (exciting in its appeal to the senses of taste, touch, smell, feel, sight) (Gifted Education, 2014).

\section{III.2.2. INTERVIEWS}

Testing variables by conducting experiments inside real organizations were not feasible because of the difficulties in applying experimental manipulations in such settings. Thus, what would be more practical and insightful was, the systematic study on an example company that is well- known for striving in its creativity and innovations. In this way, the author could gain a deep understanding on what the climate and cultures in such a company are like, and how they help bringing out the best creative abilities of its employees. Specifically, the author could determine whether competitive environment or collaborative environment, transformational leadership or transactional leadership, and pressured or relaxed atmosphere that would stimulate creative performances of employees.

The author decided to investigate the multinational technology and consulting company, IBM. IBM was chosen as it is one of the most innovative companies in the world. The company has been focusing on continuous innovation for more than a century, and has topped the annual list of U.S patent recipients for 21 consecutive years (Barinka, 2014). In 2012 and 2013 for example, IBM received 6,478 patents and 6,809 patents respectively, exceeding the combined totals of Accenture, Amazon, Apple, EMC, HP, Intel, Oracle/ SUN and Symantec (IBM Sets U.S Patent Record, 2013). As such, to study how the climates and cultures are like inside IBM would be very beneficial in answering the ultimate research question of this paper. That is, to help determine what the ideal organizational mechanisms or interventions organizations should implement, in order to bring out their employee creativity and ultimately fostering innovation in organizations.

In order to determine the cultures and climates inside IBM, specifically on the level of competitiveness or collaboration among employees, the environment regarding pressure or relaxation, and the types of leaders found in IBM, structured interviews were conducted.

There were two main participants for the interviews, which were Mr. Kurt Rindle and Mr. Nicolas Maeding from 
IBM Deutschland $\mathrm{GmbH}$ and IBM Deutschland Research \& Development $\mathrm{GmbH}$ respectively. Mr. Kurt Rindle was chosen for the interview because his responsibilities in IBM are directly involved with creativity and innovations, as he is the Cloud Technology Portfolio Leader for IBM Germany, Austria, and Switzerland. Specifically, his major responsibility was to develop and grow IBM's Cloud portfolios and solutions. His 13 years working experiences in IBM could provide valuable insights as well. The interview with Mr. Rindle was conducted on March 25, 2014 from $3.00 \mathrm{pm}-4.00 \mathrm{pm}$ via real time video chat tool called VSee.For Mr. Nicolas Maeding, he was very suitable for the interview also because his responsibilities are directly concern with creativity and innovations, as he is the Manager HD Physical Design I, working directly in the IBM Germany Lab in Böblingen. His responsibilities involve the development of various new technologies for the next IBM processors, which are ultimately applied for patents. Besides, Mr. Maeding also is the country lead for one of IBM arguably most challenging innovative internships, the IBM Extreme Blue. His 13 years of experiences working in IBM, as with the case of Mr. Rindle, might as well be very crucial for this study. For Mr. Maeding's case, due to busy schedule, the email interview was the preferred method. The author sent Mr. Maeding the exact same questions that the author asked Mr. Rindle on March 12, 2014 and got the written answers on April 16, 2014. Worth to note is that both interviewees hold the positions of managers or leaders. This adds a new perspective to what has already been done in the previous researches that involve mostly employee level participants. Managers often have a say in the organizational policy so they might provide answers as well as the reasons why.

\section{RESULTS \& ANALYSIS IV.1. RESULTS FOR HYPOTHESES}

For H1.1, H1.2, H1.3 - Creative Personality

The results of the experiment on creative personality indicated two key points. First, the majority of individuals heavily applied their past experiences and knowledge on to their creative products. That is, $79.63 \%$ of the participants imagined and drew animals on Mars with earth animal attributes, as opposed to only $20.37 \%$ of the participants who drew completely different animals. Second, individuals who drew animals on Mars with many earth animal attributes, on average, were less open to experiences, were less extraverted, but were more conscientious than the individuals who drew almost completely new creatures. Specifically, out of the total score of 5, although individuals who drew animals on mars with many earth animal attributes on average scored as high as 3.7 for openness to experiences, this was still lower than the average score of the individuals who drew animals on Mars almost without earth attributes which is 4.5. For extraversions, the differences in the scores were even higher. That is, individuals who drew animals on Mars with many earth attributes and individuals who drew completely different creatures on average scored 3.5 and 4.7 respectively. For conscientiousness, the results showed that individuals who rely a lot on their past experiences and knowledge scored very high, which is an average of 4.0, while individuals who applied less of such knowledge on average scored very low of only 2.9 .

\section{For H2 - Moods \& Emotions}

The results of the experiment on break and no break in between creative task showed that, on average, only the minority 
of individuals performed better on creative tasks when they took a break. Specifically, results indicated that only 13 out of 54 participants, or $24.07 \%$ performed better when they took such break. In fact, surprisingly, the majority of participants performed worse when they took a break than when they were not. Particularly, their creative scores dropped from an average of 21.93 points to 20.22 points, or a decreased of $7.80 \%$.

\section{For H3 - Group Diversity}

The results of the experiment on group diversity showed that, on average, groups with heterogeneous members, measured in terms of educational background, performed better on creativity tasks than groups with homogenous members. Specifically, the average creativity score of heterogeneous groups was 20.56 points, as opposed to only 16.33 points for homogenous groups. On average, this represents $25.90 \%$ improvement in creative performances for the heterogeneous groups.

\section{For H4 - Group Membership Change}

The results of the experiment on group membership change showed that, out of the 18 groups, on average, 15 groups performed better when they experienced group membership change. This represented $83.33 \%$. These groups scored higher in round 3 and 4 where they lost and gained new members, in comparison to round 1 and 2 where the movements of members were static. Specifically, the average creativity scores when groups experienced membership change was 19.0 points, as opposed to only 14.9 points when they experienced no membership changes. On average, this represented 27.52\% improvement in creative performances.

\section{For H5 - Idea Generation: Brainstorming}

The results of the experiment on brainstorming showed that, all 18 groups participated in this experiment performed much better in creative thinking tasks when their members worked on these creative tasks individually, than when they worked in their respective groups. The average creativity scores for group brainstorming was only 14.90 points, as opposed to 32.52 points when the scores of individual brainstorming were pooled together. Expressed differently, when individuals worked on the creative tasks on their own and combined their efforts later, after elimination of redundant ideas, they performed $118.26 \%$ better than when they brainstormed in a group setting.

\section{For H6 - Leadership Style}

Examination of the common leadership styles within one of the world's most innovative companies, IBM, indicated two major themes. First, transactional leadership, which commonly involves leading through reinforcement, reward and punishment based system to drive employees to achieve targets, was believed to be beneficial for the likes of project execution and functions such as sales and distributions. As highlighted by Mr. Nicolas Maeding, transactional leadership is important in critical situations or situations involving rules and regulations. This is because, transactional behaviors of the leaders often leads to micromanagement and a focus to deliver predictable and fast solutions, to get things done that is. As for Mr. Kurt Rindle, transactional leadership style only make sense for the low skill, simple, and repetitive tasks such as ones found in the factories or assembly lines in the manufacturing industry. The reward and punishment system works well with 
these production line workers as they have some clearly defined repetitive tasks and goals. That is, the more parts are being assembled into final products, or the more parts are being produced, the more rewards they will receive. In contrast, the second major finding from the study of leadership style in IBM indicated that, in the lab environments or other innovative functions and departments, transformational leadership style seems to bring out the best creative potential of the individuals. Transformational leadership tends to involve the efforts of the leaders to change or transform their followers' needs and redirect their thinking, motivating and inspiring them through vision, and a sense of purpose and excitement. These leadership behaviors were evident among the two innovative leaders of IBM in question. For Mr. Maeding, his general attitude as a leader is transformational in the sense that his horizon is on the transformation of the team and the larger coordination, rather than the short term project execution or management focus. Specifically, Mr. Maeding always look for opportunities to drive and promote topics "outside the box", instead of looking for more responsibilities in a given project or the chance of being appointed to a greater role with more "formal" responsibilities. As for Mr. Rindle, he sees himself as a transformational leader who always inspires and motivates others, leading by examples, and constantly anticipating and encouraging change. For instance, Mr. Rindle lead by example through taking the time to work with his followers, show them how he would do things, how he would have solve the problems, then hand over the task to them step by step. As with one of his new subordinates as an example, Mr. Rindle went through the project materials with this subordinate, went to the first customer engagement together and showed how he would do it, then gave the subordinate the opportunity and room to learn and work on her own. In addition, Mr. Rindle always anticipated changes for the better. He explicitly expressed his belief that one can always improve things, that he or she does not have to wait until "things get broken". Mr. Rindle strongly believes that these transformational beliefs and attitudes of the leaders have potential in driving creative potentials of the employees because it leaves room for ideas. Like in the IBM innovative departments, Mr. Rindle described that people there are free to think and to explore whatever they wanted. There is hardly any tight control or tight management, telling people exactly how to do their jobs. That is how people invented things in IBM, invention through freedom that is. Mr. Maeding also stressed the importance of transformational leadership style in driving creativity through the chance to experience "trial and error", which is hardly possible with transactional leaders. Creativity is something that is not guaranteed, as Mr. Maeding mentioned, it may benefit from wild ideas and the ability to allow detours and partial failure. In addition, worth to note is that, transformational leaderships is not only valued at the managerial positions in IBM, but also at the very top management level as well. According to Mr. Maeding, higher management in IBM tends to communicate and act transformative, embracing changes within the company. For example, as highlighted by Mr. Rindle, everyone in IBM was asked to always reinvent the things they do. This has been communicated through one of the IBM's three core values, which is "Innovation that matters, for our company and for the world". Creating original ideas is at the heart of IBM. Top Management drives and treasures this no less than something else. In essence, what has been illustrated here is that, IBM, one of the 
most innovative companies in the world, tends to adopt a more transformational style of leadership, which is evident through the likes of managers and leaders' behaviors and attitudes, top management's beliefs, as well as the company's core value, where every single employee, if regarded themselves as an IBMer, is ought to follow.

\section{For H7 - Competitive Environment}

With the number of patents received second to none, it is very interesting to investigate how the environment is like inside IBM. Specifically, it would be beneficial to learn whether the atmosphere inside this highly innovative company is more of a competitive one, or more of a collaborative one. When questioned about their opinions or personal experiences, both Mr. Maeding and Mr. Rindle described IBM as having an organizational culture that is more collaborative than competitive. To be precise, Mr. Rindle mentioned, "I never experienced any competitiveness inside the company". As for Mr. Maeding, he clearly pointed out that such collaborative atmosphere can be seen not only in Germany, but also across all IBM global locations. In addition, Mr. Maeding stressed that, collaborative atmosphere is not only seen in his specific department which directly involves innovations, but also seen across other non R\&D departments inside IBM as well. According to him, a number of things make the atmosphere inside IBM more collaborative. First, there is an open door policy where everybody can ask or stop by anybody. This reduces obstacles in communication and thus encourages collaboration. Second, instead of competing against one another, the culture inside IBM is to collectively resolve issues and not finger-pointing to the originator. The root causes of the problems will be analyzed and shared among teams as lessons learn to avoid future repetition. Third, Mr. Maeding described the collaborative atmosphere inside IBM as one that is uncommon to exclude individuals or prevent them from interacting and discussing with, for example, higher technical leaders. Specifically, there is an "innovation development team", a group of senior inventors who support engineers by reviewing their novel ideas and provide support in the further evolution of their ideas. In fact, IBM has implemented various other mechanisms and tools, in an effort to promote collaborations among its employees. For instance, both Mr. Maeding and Mr. Rindle mentioned about the extensive promotions and uses of the "IBM Social Collaboration Tools", such as IBM Lotus Sametime, and the newly implemented program called 139 . IBM Lotus Sametime involves instant messaging, online meetings, videos, and data for real-time business collaborations. With just a simple click, IBM employees all over the world are instantly connected to one another. For 139, as Mr. Rindle had described, it is like having facebook or twitter within the company. It is like an online platform, and more, where employees can engage and collaborate. That is, they gathered to tell what they have achieved, with whom, how they work, along the 1 main purpose of the company which is "to be essential", 3 values which are "Dedication to every client's success", "Innovation that matters, for our company and for the world", and "Trust and personal responsibility in all relationships", and 9 practices such as " treasure wild ducks", "restlessly reinvent our company and ourselves, and " dare to create original ideas". As such, this 139 social collaboration tool does not set up just for employees to collaborate, but to collaborate with special focus on 
innovations. In addition to such tools, what the author found surprising was the responses from Mr. Rindle regarding IBM's promotion of collaborative efforts through shortage in resources. That is, IBM puts shortage of resources on, for example, people and time, so that people are in a situation where no one is competing for the same job or the same role, and there is always more work than one can deal with. Under such environment, people tend to work together, helping one another to achieve the goals that are difficult to achieve when collaboration is not there. As such, it seems that there is an ongoing effort within IBM to promote collaborations among its employees. How collaborations help creativity, then, is the next interesting question. According to Mr. Rindle, with the matrix organizational nature of IBM where people are dispersed in different locations across the globe, the social collaboration tool helps immensely. Ideas can be shared in the blogs without the obstacle from locational bases and people can search and find what other people think. Ideas thus can grow out of others' ideas. As for Mr. Maeding, the opportunity to discuss ideas with seniors or distinguished engineers enables the younger innovators to learn, and thus drives their creative abilities. Also because these round table discussions are neither formally appointed nor given, but rather evolved based on the interest of the group and the new ideas individuals bring to the table, creativity is likely to emerge. Thus, IBM actually does not force collaboration but indirectly influencing it. Creativity is hardly a result of forced actions. In addition to the discussion on collaboration, Mr. Maeding has given some insights regarding competitive environments within IBM. He mentioned that, when recognition and compensation is involved, competitiveness among employees is required. Specifically, Mr.
Maeding pointed out that, this sometimes has negative impacts on the collaborative development culture. That is, individual rewards such as outstanding technical achievement lead to high competition among employees so there will be less collaboration among them, and as a result may be less creative.

The results of the experiments on competitive environment supported the information obtained from the interviews. As illustrated below in table 8 below, when a sense of competition is induced to the participants, their creative performances tend to decrease (Examples of participants' work on this exercise are shown in Appendix G). Specifically, out of the total 11 points, when high level of competitiveness was induced, the creative scores on average were reduced from 5.48 to 3.33 . This represented a decreased of $39.23 \%$.

\section{For H8 - Environment of Stress \& Pressure}

It is interesting to study how the working atmosphere in terms of stress is like in IBM, especially in the departments dealing directly with innovations. Do professionals in these innovative departments work well under a highstress environment, or they prosper under a more relaxed one. The answer to such question may seem obvious, but the findings from the interviews in fact suggested otherwise. That is, in order for ones to reach their full creative potentials, it is not about environment that is relaxed or not relaxed, pressured or no pressured, but it's more about flexibility and freedom. As Mr. Maeding has importantly pointed out, "creativity cannot be unified". In order to bring out the best creative abilities of the individuals, the efforts made by organizations must be tailored to each individual's needs. That 
is, some individuals prefer brainstorming, others generate best ideas in a follow-up of presentations, while some others had their best ideas when they were walking through the forest during the lunch breaks. Without such flexibility and freedom, Mr. Maeding strongly believes that the innovation rate will decrease rapidly. As such, it is a challenge for the leaders to determine the individual's best mode for creativity, and enable it as much as possible, or request support from higher management. As for Mr. Rindle, freedom is also the key to creativity and innovations. He believes that creativity needs room and freedom, for example, people should work only half the time and leaves the rest of the time open to new ideas. Just trying to get things done does not help creativity at all. Mr. Rindle again referred back to the example of repetitive tasks like in the car manufacturing facility. He suggested that, inducing pressures seems to work well for such piece rate based jobs as the more one gets done in less time, the better he or she will be paid. In contrast, for creative thinking, ones must be able to have their minds roam free, to have the freedom to think, to be happy in what they do. As such, Mr. Rindle suggested that, the challenge for the organization is to have the right people in the right job. Only then ones will be creative. In fact, what has been stressed a number of times by both Mr. Maeding and Mr. Rindle was that, instead of creating a relaxed, low- pressured work environment for employees, what organizations should do in order to bring out the best creative potentials of their employees is to provide "work- life integration", or the opportunity for employees to blend and integrate their work and private life together in order to make both work. This is exactly what IBM provides to their employees. For example, both Mr. Rindle and Maeding mentioned about flexible working time and place. IBM employees are free to choose where to work, and when to work. For instance, one does not have to start working early in the morning but can start late in the afternoon and work until night, as long as the works align with their customers. Employees, furthermore, may also choose to work at home, if that suits them more. In addition to such freedom of choice, Mr. Rindle mentioned about other various efforts made by IBM in order to make the workplace more of a work-life integrated one. For instance, in some IBM locations, there are kindergartens where IBM employees can bring their children to work. Employees can also order fresh food from the supermarket, and their ordered food will be delivered and stored in the freezer lockers where they can pick up when they go home. There is also a dry cleaning service where employees can drop their clothes and bring them back the next morning. As for Mr. Maeding, he specifically mentioned about the organization's efforts to make employees feel at home, through the likes coffee carts and corners, as well as table soccer for employees to enjoy. Thus, as has been stressed by both Mr. Maeding and Mr. Rindle, when employees do not have to worry about their private lives, then they are free to explore and be creative. Just like what Mr. Maeding pointed out, although the things to make employees more relax are not implemented in his department, a static rate of patents and innovations were evident anyhow, suggesting that relaxation is not what makes ones creative, but the freedom, the flexibility, and the work-life integration that are.

The experiments on break and no break conducted at the individual level which was mentioned earlier, supported these points of view by Mr. Maeding and Mr. Rindle. Specifically, the experiment indicated that, providing a break during 
creative task, in an attempt to make participants feel more relaxed, did not actually help them to be more creative. In fact, results had shown a slight decreased in their creative performances.

\section{IV.2. SIGNIFICANCE TESTING FOR QUANTITATIVE DATA}

In order to assess whether the observed differences between each set of samples were statistically significant, in other words, whether there was a measurable probability that the sample statistics were good estimates of the population parameters, significance tests were conducted.

As illustrated in table 2 below, the p-values were $<0.05$ for all hypotheses except for hypothesis

3. Therefore, the null hypotheses for H1.1, $\mathrm{H} 1.2, \mathrm{H} 1.3, \mathrm{H} 2, \mathrm{H} 4, \mathrm{H} 5$, and $\mathrm{H} 7$ will be rejected. However, for hypothesis 3, with the current data there was not enough evidence to reject the null hypothesis. Therefore, with $95 \%$ confidence, the author concludes the following: Individuals who did not heavily rely on their past experiences and knowledge, on average scored statistically significantly higher on openness to experience and extraversions, and scored statistically significantly lower on conscientiousness, than those individuals who relied more on their past experiences and knowledge. When groups experienced team membership changes, their average creative scores were statistically significantly higher than when they did not experience such changes. For brainstorming, when groups had their members individually brainstormed as opposed to brainstormed together, their average creativity scores were statistically significantly higher. On the other hand, when individuals took a break in between creative task, their average creative scores were statistically significantly lower than when they did not take such a break. Groups that performed creative task under competitive environment also had statistically significantly lower average creative scores than those in the control condition. Last but not least, the results of the significance test revealed no statistically significant differences in the average creative scores of heterogeneous groups and homogenous groups. Specifically, the higher average creative score for the heterogeneous groups was not statistically significant.

Table 2. Significance Test Results

\begin{tabular}{cccccccccccc}
\hline $\begin{array}{c}\text { Hypo } \\
\text { \# }\end{array}$ & $\begin{array}{c}\text { Mean } \\
1\end{array}$ & $\begin{array}{c}\text { Mean } \\
\text { Var }\end{array}$ & $\begin{array}{c}\text { Var } \\
1\end{array}$ & $\begin{array}{c}\mathrm{N} \\
\mathbf{1}\end{array}$ & $\begin{array}{c}\mathrm{N} \\
2\end{array}$ & df $\mathrm{t}$-Stats & t-Critical & P-Value & $\begin{array}{c}\text { Decision } \\
\text { Reject Ho }\end{array}$ \\
\hline 1.1 & 4.5 & 3.7 & 0.12 & 0.09 & 11 & 43 & 14 & 6.42 & 1.76 & 0.000000795 & Yes \\
\hline 1.2 & 4.7 & 3.5 & 0.17 & 0.13 & 11 & 43 & 14 & 8.33 & 1.76 & 0.000000425 & Yes \\
1.3 & 2.9 & 4.0 & 0.29 & 0.25 & 11 & 43 & 14 & -6.47 & -1.75 & 0.00000525 & Yes \\
\hline 2 & 20.22 & 21.93 & 96.47 & 93.33 & 54 & 54 & 53 & -3.23 & -1.67 & 0.001053 & Yes \\
\hline 3 & 20.56 & 16.33 & 28.11 & 34.72 & 9 & 9 & 16 & 1.60 & 1.75 & 0.064805 & No \\
\hline 4 & 19.04 & 14.90 & 13.90 & 18.25 & 18 & 18 & 17 & 4.78 & 1.74 & 0.0000871 & Yes \\
\hline 5 & 32.52 & 14.90 & 71.35 & 18.25 & 18 & 18 & 17 & 8.39 & 1.74 & 0.0000000944 & Yes \\
\hline 7 & 3.33 & 5.48 & 1.83 & 5.59 & 9 & 9 & 13 & -2.37 & -1.77 & 0.017099 & Yes \\
\hline
\end{tabular}

\section{IV.3. ANALYZING QUALITATIVE DATA}

Unlike quantitative data from the experiments, the data to be analyzed from the interviews are text rather than numbers. The key to analyzing textual data is to look for significant patterns of ideas or repeated themes (Schutt, 2011)

It was hypothesized that a very innovative company like IBM should have leaders who manifest transformational behaviors. From the analysis of the interview answers, three themes are common among the two interviewees, and these support the hypothesis set earlier. First, both interviewees repeatedly mentioned about how transformational leadership bring out the best creative potential of individuals as opposed to transactional leadership. Specifically, they pointed out that transactional leadership suits 
more to repetitive, disciplinary tasks while transformational leadership suits more to more complex, creative problem solving. Second, it is evident from the interviews that managers, leaders, as well as top management in IBM embrace and lead with transformational mindsets. They always challenging the status quo, asking each and every employee to reinvent everything they do. Third, both interviewees described the environment inside IBM as one that is transformational in nature, that there is hardly any tight control but a lot of freedom for employees to think and explore their ideas. Therefore, if this is what the environment is like inside a very innovative company, then transformational leadership must somehow be beneficial to creative thinking.

It was hypothesized that organizations that encourage competitions among employees are less likely to be creative than organizations that encourage collaboration. From the interview answers, three common themes among the two interviewees emerged. That is, first, the environment inside IBM is definitely one that is very collaborative. Competition among employees was discouraged as they are believed to negatively impact collaboration and in turn creativity. Second, it is very clear from the interviews that IBM currently has many collaboration tools for their employees. Employees who are dispersed across the globe can connect and share their ideas with ease. Third, both interviewees have mentioned repeatedly how they believe collaborations would bring out the best creative potential of the employees. For instance, ideas of one can grow out from the ideas of others. Therefore, the company with countless patents like IBM has the environment of collaboration rather than competition. Although not directly indicated, competitive environment seems to not have advantageous effects but even detrimental.

It was hypothesized that pressured environment is more likely to hinder organizational creativity as opposed to relaxed environment. Two common themes emerge from the two interviewees when question about this. First, surprisingly, both agreed that it's not about relaxed or pressured environment that makes individuals creative, but flexibility and freedom that are required. Specifically, they mentioned in detailed how "work-life integration" would help employees to be more creative. That is, when employees do not have to worry about their work while at home, and when they don't have to worry about their private lives while at work, their minds are ready to roam free. Second, not only employees inside IBM crave for freedom and flexibility, the company actually provides them. They provide their employees with a lot of freedom, flexibility, and of course opportunity to integrate their work and private lives. IBM employees are free to bring their kids to work, can choose their working time, as well as having groceries personally delivered to work. Therefore, what can be concluded is that, an innovative company like IBM does not emphasize relaxed or pressured environment, but one that is flexible and provides a lot of freedom.

The themes identified from the interviews based on each hypothesis are presented in table 3 below. In conclusion, hypothesis 6 and 7 will not be rejected, but hypothesis 8 is. 
Table 3: Summary of Common Themes from Qualitative Hypotheses

\begin{tabular}{|c|c|c|}
\hline Hypothesis\# & Common Themes & Decisior \\
\hline 6 & $\begin{array}{ll}\checkmark & \text { Transactional leadership suits more to repetitive, disciplinary tasks } \\
\checkmark & \text { Transiormational leadership brings out the bestcreativepotential ofindividuals } \\
\checkmark & \text { Transformational leadership are evident amongnoton y leaders and managers of } \\
\text { IBM, but also among top management and the company core values } \\
\checkmark \quad \text { Environmentinsidel BM i sone thatis transformationalin nature, that there is } \\
\text { hardly any tight control but a lot of freedom to think }\end{array}$ & Accept \\
\hline 7 & $\begin{array}{l}\checkmark \quad \text { Environmentinside IBM is one thatis very collaborative, as opposed to } \\
\text { compettive } \\
\checkmark \quad \text { Many collaboration tools are available for employees } \\
\checkmark \quad \text { Collaborations bring out the best creative potential of individuals }\end{array}$ & Accept \\
\hline 8 & $\begin{array}{l}\text { Employees creative abilities arenotenhancethrougharelaxed, lesspressured } \\
\text { environment, but through flexbility and freedom. I's more about "work-life" } \\
\text { integration } \\
\text { IBMcurrentyprovidedalotoffreedom, fexibility andwork-lifeintegration } \\
\text { supports for their employees }\end{array}$ & Reject \\
\hline
\end{tabular}

\section{DISCUSSION \&}

\section{RECOMMENDATIONS}

The present study was designed to determine the ideal mechanisms or interventions organizations should implement, that would best target the brain, bringing out its creativity and ultimately fostering innovation in organizations. In order to determine such brain-targeted organizational strategies, the author examined various organizational creativity variables, based on the fundamental understanding on the nature of the human brains.

The study provides a number of important implications for driving organizational creativity. First, organizations can stimulate creativity inside the workplace by recruiting the right people to work on the creative projects. Specifically, it is important for organizations to assign people with the right personalities to these creative tasks that can be unique and demanding. To accomplish this, the right tools as well as personnel are very much needed. That is, the human resource department plays an integral role in identifying and recruiting individuals from outside, while managers and leaders may recognize individuals with suitable personalities from the inside. For tools to assess one's personality, as with the present study, organizations may adopt the widely accepted and utilized standard personality test, the Big Five Inventory (BFI), which is a self-report inventory designed to measure the Big Five Personality dimensions. Since this is a self-report measure, organizations may want to add accuracy to its analysis by looking for help from personality experts. Organizations that are able to identify the most suitable individuals for their creative projects should be able to achieve greater creativity because individuals are now working on projects that are right for them, and even naturally interesting to them. Simply stated, personality fit is important as it may have substantial effect on job satisfactions and job performances. So, what kinds of personality organizations should look for is the next interesting question. This is where the present research can contribute more or less. That is, the findings of the present study indicated three personalities important for creativity. These include openness to experience, extraversions, and unconscientiousness. Specifically, study has found that individuals who relied less on their knowledge and past experiences for their creative productions, tend to be ones with such personalities, as opposed to others who relied heavily on their past experiences and knowledge. Since the projection of knowledge and past experiences into new solutions lead to ideas of lower originality, individuals who did not do so are said to be more creative and tend to produce more original ideas. Therefore, what this could mean is, experts who possess a lot of domain knowledge and experiences do not necessary have the most creative insights. As have shown, with experience comes rigidity. Experts have so much invested in their own fields and theories 
and point of view that they tend to defend them and become increasingly rigid. Therefore, it may also more beneficial to recruit the inexperienced individuals who may bring about new and different perspectives. Consequently, organizations do not have to spend a whole lot of money in hiring very experienced individuals to work on innovative projects. The observed relationship between the three personalities of individuals and their tendency to rely on past experiences and knowledge can possibly be explained by how the human brain works. As has been thoroughly discussed in the literature review section, the human brain tends to always find ways to save energy, for example, by making predictions about the incoming stimuli or filter out information that it thinks are irrelevant based on the past experiences and knowledge. However, individuals who are more open to experiences and extraverted, in other words individuals who are curious, strive to try new things, sociable, more open to the outside world, are not limited by such past experiences and knowledge and thus interfere the brain's energy conservation system. This is because such personality traits make them embrace and open to more possibilities, more stimuli from the outside world that can be very original and thus important for creativity which requires novel combinations of ideas. Neurally speaking, for example, an introvert is believed to be easily cortically aroused from the outside social interaction events. This cortical arousal is possible via the narrowing of neural networks which is achieved through filtering out irrelevant stimuli using past experiences and knowledge. However, for an extravert, he or she is usually energized by being around others which leads to lower cortical arousal level and thus accepted more outside stimuli. In addition, the research on brain science suggested that the human brain always search for certainty, always try to predict thing. What this means, then, is knowledge is very important for the brain as the lack of knowledge could consequently lead to uncertainty. Therefore, individuals who are less conscientious, or in other words individuals who values less planning but more uncertainty, are likely to rely less on their knowledge and past experiences. Expressed differently, individuals who are less conscientious tend to be able to escape from the conventional ways of thinking and consequently develop ideas of greater originality. Although to the author's best knowledge, currently there is no research specifically investigating individuals' tendency to rely on their past experiences and knowledge and their personalities, the findings from the present study are still consistent with the earlier studies that examined individuals' personalities against other aspects of creativity. For example, several studies suggested that openness to experience, extraversions, and unconscientiousness personality traits favor the development and expression of new ideas (Dollinger \& Clancy, 1993; King, Walker, \& Broyles, 1996; Feist,1998; Wolfradt \& Pretz, 2001; Batey, Chamorro-Premuzic, \& Furnham, 2009). Particularly, some studies proposed that close-minded individuals have the desire for cognitive closure. That is, the desire for definite and firm answers rather than ambiguity. So, they tend to reach quick closure on an issue, and prefer to maintain such closure after it has been reached. Therefore, this may negatively affect creative thinking process which can be time consuming and involve uncertainty and indefinite solutions (Webster \& Kruglanski, 1998). For conscientiousness, several researches have been proposing that individuals who are more conscientious may have personal need for structure (PNS). PNS refers 
to a chronic aversion to ill-structured situations and a longing for certainty and predictability. As such, researchers expect PNS to be negatively related to creativity because creativity is something that involved uncertainty and derived from the unpredictable. Although very interesting, this specific area of research needed more supports (Thompson, Naccarato, Parker, \& Moskowitz, 2001).

The second major implication is for the determination of ideal idea generation strategy that would lead to highest creative performances and outcomes. To stimulate the generation of the best creative ideas, the results of this study suggested that nominal brainstorming, or in other words individual brainstorming, should be preferred over group brainstorming. Specifically, as hypothesized the results showed that the solutions generated from individuals who worked separately but later combined their ideas were on average significantly higher than when they worked together in groups. This phenomenon can be further explained using biological reasons. That is, group brainstorming enables the brain to initiate its energy saving mode, resorting to the least cognitively demanding path, by relying on the ideas from others or just conform to the ideas of others. This kind of environment may favor the brain to conserve its energy, however, it does not favor creative thinking which involves highly cognitive processes to produce something new and different. On the other hand, when individuals brainstormed on their own, their brains have no choice but to try and work on producing creative solutions since there are no others for them to rely on. The results of this idea generation strategy investigation are consistent with considerable amount of earlier organizational creativity work where individual brainstorming method tends to outperformed group brainstorming method due to presumed reasons such as elimination of process loss like free ridings and topic fixation (Taylor, Berry, \& Block, 1958; Dunnette, Campbell, \& Jaastad, 1963; Diehl \& Stroebe, 1987). Therefore, although contrary to the common belief of practitioners, organizations should encourage employees to work on their own while providing them with necessary supports and adequate resources. That is, organizations should create an environment where employees believe they can strive on the creative tasks on their own, but not entirely excluded from the guidance and supports from others. However, practically, this really depends on the nature of the creative problem as well. When the creative problems are very complex in nature, brainstorming in the form of group may still be beneficial. As such, what the organizations should do in order to take the full benefits of team brainstorming and eliminate its disadvantages is to use technology to facilitate idea generation, the implementation of electronic brainstorming that is. This brainstorming method involves team members to interact and exchange ideas by typing out their ideas into their computer and each idea is immediately displayed on the other participants. Therefore, who are contributing what and how much are clearly displayed. This, thus, should help prevent process loss due to free riding as the brains are now forced to think harder. Moreover, electronic barnstorming help reduce process loss as it enables members of the team to simultaneously introduce ideas without having to wait for their turns. In fact, there are some researchers who investigated the impact of such electronic method on the creative performance of the teams and found no productivity loss occurred (Dennis \& Valacich, 1993). 
The third important implication for stimulating employee creativity is organizations should encourage and introduce membership changes in teams. That is, the membership of the creative projects should not be permanently static but rather includes some new comers joining and some existing members leaving. This may sound simple but practically may be hard to execute and achieve the desired effect without special care. It's the job of the organizations to ensure smooth transitions, for example by getting the newcomers up to speed of the team as fast as they can and providing necessary supports to them. This implication on the positive effects of team membership change is supported by the results of this research. That is, as hypothesized, when groups experienced team membership changes, they were on average more creative than when they did not experience such changes. To provide biological explanations on this, the insights on how the human brain really functions are very much valuable. Brain research has shown that the human brain embraces certainty as well as social qualities such as belongingness above all else. Therefore, in its power it is likely to do whatever it can to keep such a low risk, high conformity status, which can be detrimental to creative thinking that tends to involves something different, something novel. Since membership change involve experiencing unfamiliarity through the introduction of new members, such change should stimulate creativity by forcing the brain out of its comfort zone, out of its usual path-of-least-resistance and social conformity, and more into forming new neural pathways to deal with such unfamiliar situations. These results are in line with the other several previous studies. For instance, earlier organizational creativity studies found that teams that have static membership tend to be less creative as they were more bias toward discussion common information (Janis, 1972; Katz, 1982). On the other hand, several researchers found that membership change enhances groups' creative potential because new members introduced to the team bring new information, perspective, and ideas which may broaden and diversified the knowledge base of the team (Choi \& Levine, 2004). Although results on group membership changes were as hypothesized, contrary to expectations, the study did not find significant differences between the creative productions of groups with homogenous members and groups with heterogeneous members, although they were based on the same biological explanations. It seems possible that these surprising results may be due to relatively low degree of diversity in heterogeneous sampled teams. As the present study adopted the design of heterogeneous team consisting of two members of the same education background and one member of a different education background from the two, this mix may not be diverse and different enough from the homogenous teams to have an impact on the overall creativity of the team. Therefore, future researches may tackle this and set up teams of three members with each of them having different educational background. Also because the sample size of this experiment is relatively low, more participants may be needed to verify the results.

The fourth and final major implication from the results of this study is regarding the designs of neurobiological suitable working environments. That is, creating environments that are healthy for brains in business context, the environments that drive the brains to operate at their maximum creative ability. The track records of failed efforts to stimulate 
creative behaviors in employees have led many managers and leaders to conclude that human nature is simply unmanageable. However, understanding biological basis of human can help leaders who are trying to implement such a large-scale change. Specifically, neuroscience has discovered that the human brain is actually highly plastic, in other words it is an everchanging organism. This means that the neural connections can be reformed, new behaviors can be learned, and even the most difficult to change behaviors can be modified at any age (Rock, 2009). These neural connections can grow and shrink according to how much individuals use the pathways. Therefore, the insight on such neuroplasticity suggests that the brain can and does change, but the environment must be suitable. This is because the environments cause the brain and the neurons to fire in specific patterns (Ibid). It affects the behaviors and experiences that forms the connections in the brain that is. As such, the major implication from this is that, organizations must utilize the right stimuli, the right environments for the brain to achieve its best creative potentials. Neuronally speaking, it is the external stimuli which are crucially important in determining what neuronal network connections are made. The first brain-targeted recommendation based on the results of the present study is to create organizational environment with transformational leadership influences. That is, organizations should have leaders who constantly encouraging employees to question assumptions, to take new tasks and challenges. Leaders and managers should create an environment of trust, where they encourage employees to tackle the problems and trust them to find and create their own great creative solutions. Furthermore, leaders and managers should act transformative by developing a positive mistake culture inside the organizations. There tend to always be individuals who fear of saying something they believe are nonsense and make them look bad. If employees are punished for their mistakes as has been observed in the transactional leadership style, this may block them from expressing their ideas and eventually block creative thinking to rise in the workplace. The mentioned transformative leaders' behaviors are very much evident in the managers, leaders, as well as top management of IBM where the rate of patents has been increasing rapidly year by year. IBM in addition promotes innovation as its organizational value. They did not just relying on hiring creative people, but also placed creative and even average individuals into creative culture. Such an environment is well recommended for others who wish to develop innovative organizations, and follow suit. The postulation why transformative behaviors of leaders can be more conductive to employee creativity as opposed to transactional behaviors can be explained again using biology. As has been stressed several times, there is a strong tendency of the brain to resort to what it already knows, to what it believes will be the least against the common. However, transformational leadership is likely to overcome such nature of the brain and bring out the best creative potential of the employees by emphasizing change, encouraging employees to achieve extraordinary outcomes. These activate neuronal networks that would otherwise remain passive. For transactional leadership, fear and stress causes distortions in the brain and as a result activates the fight or flight response which diverts the brain energy from solving creative problems to focus on survival instead. The present findings regarding which leadership style is best for stimulating employee creativity are 
also consistent with several previous researches. For instance, there are studies which found transformational leadership as a successful mechanism to increase creativity and innovation in organizations (Howell \& Higgins, 1990; Khan, Rehman, \& Fatima, 2009; Gong, Huang, \& Farh, 2009; Alarifi \& Althonayan, 2013). The second brain-targeted recommendation based on the results of the present study is for organizations to create an environment that values collaboration as opposed to competition among employees. These employees should be allowed to focus on their creative problem solving tasks, and not worry about competing against each other. A healthy collaborative culture is one in which employees feel free to share their ideas, opinions, as well as criticism. Such an environment is well reflected inside IBM. The company represents a great example in promoting collaborative efforts among employees. That is, it uses technology as a mean to collaborate and share knowledge. Collaboration drives creativity and social media and conferencing technologies can help bring people together for that collaboration, which is extremely important for the business world today where everyone tends to be dispersed around the world. To be exact, IBM uses an online platform called 139 where employees can share and discuss their great ideas. Such tool is linked to the company's core values, which is again a great way to explicitly say to each and every one inside the company that collaboration and innovations are valuable. Therefore, for creativity to flourish organizations should not just create a collaborative environment, but organizations should make its importance clear to everyone. Neurally speaking, organizations should emphasizes collaboration instead of competition among employees because under competitive situations, individuals may experience stress or pressure which tends to lead to high arousal states where neural networks become narrowed and thus restricted the breadth of activated concept representations stored in such neural networks. As a result, this may suppress the emergence of remote associations, the important ingredients for creative solutions. The results of this study in fact are consistent with the few earlier observations. For example, several studies have found that when competitions were induced, lower creative performance seemed to be presented (Amabile, 1982; Tjosvold, Tang, \& West, 2004). The third and final recommendation for braintargeted environment is, instead of spending tremendous amount of money in creating relaxed atmosphere for employees, what organizations should do to stimulate their creativity is providing them with freedom and flexibility to work. This is because, relaxed atmosphere does not always lead to improved creativity. As has been surprisingly discovered in this study, individuals did not perform better in creative tasks when they took a break compared to when they did not take such a break. In fact, they actually performed better when they did not take a break. Although the differences in their raw creative score were minimal, it is statistically significant. Like in IBM, the organization is not emphasizing such relaxed atmosphere but rather on providing their employees with maximum autonomy to carry out their jobs. Overly strict management of personnel is hardly seen in the company like IBM. This is where IBM believes creativity can develop successfully. The company, for example, providing freedom to employees regarding the time period to work in a day, the freedom to choose, to explore and think without tight control by managers and leaders breathing down their necks. In fact, IBM went a bit further by 
implementing work-life integration atmosphere instead of relaxed atmosphere, for instance by proving services such as on site food delivery and dry cleaning, playgrounds for their children etc. Biologically speaking, this makes much sense as well since when employees do not have to worry about their private lives while working, and do not have to worry about their work while at home, means that they can be less stressful and as a result having lower cortical arousal due to lower level of norepinephrine being released which widened the spread of neural networks that are conductive for creative thinking.

\section{FUTURE RESEARCH OUTLOOK}

The suggestions for further research are based on two main points: parts of the work which needed further improvements due to resources constraints or problems encountered, and new areas of investigation implied by developments of the present research.

The limitations of this study provide a number of insights for future research. First, the scholars who are not constrained by time and resources should consider adopting a longitudinal study in order to thoroughly investigate the stability or changes of the interested variables. However, if time and resources do not permit, especially for team activities such as team member change, the researcher may consider using teams whose members are already part of the same group prior to the study. For example, these individuals might be working on a certain projects together at the universities or their companies. Second, researchers who have the required time and resources should aim for a larger sample size, as well as the true targeted populations which are employees currently working in organizations, to ensure the true representations of the interested populations and the generalizability of the findings. Third, the researchers should use the original TTCT to prevent any discrepancy in the tasks employed as well as to use professional or expert creativity judges to prevent any of the misinterpretations of test results. Fourth, the researchers should allow themselves enough time to research and gain an in-depth understanding on the two complicated and complex field of studies, which are creativity in organization and neuroscience of creativity. Without a firm foundation of these two areas, it will be difficult to develop hypotheses that are both addressing the existing gaps and contribute to the novel areas of research. In addition, as previously mentioned, future researchers may want to further investigate the group diversity variable. Specifically, researchers should make sure that the degree of diversity is sufficient for the differences between homogeneous and heterogeneous groups, if any, to emerge. Also, sample size should be larger than currently adopted. The last thing the author would like to highlight is, although the author mainly adopted lab experiment approaches, this is not to say that field research is completely useless. To fully understand such a complex phenomenon like organizational creativity, it requires insights from all perspectives. Lab and field experiments each add unique and complementary insights. Therefore, individuals who are well equipped with the required resources may consider testing the author's hypotheses under the natural business environments as well. However, as mentioned before, the study must be well designed to address numerous factors at the natural environments which are hard to control and may affect the results of the study. The recommendations that are based from the development of the present 
research, the author briefly describes them next. First, although the present study found that the nature or properties the brain are somewhat similar across individuals, future researchers may be interested to examine whether there are any significant differences between the structures and functions of the male and female brains, or between the young and the older brain that might constitute differences in their behaviors and consequently demanding different treatments. Second, future researchers may consider studying creativity in other functions of organizations and not limited to departments directly involved with innovations as in the present study. It is often thought that creativity is a serious analytical task restrained to certain disciplines such as Research \& Development. However, in reality creativity for other departments like finance, marketing, or HR are very much needed to drive innovations in their processes and every day work as well. Third, the present study provides insights into how to stimulate creative ideas in organizations, but it did not focus on ideas implementation. Therefore, future research may be interested in investigating how to best translate creative ideas into innovative actions and results. This translation processes is as important for the organizations that want to be more innovative as the idea generation processes, therefore it should not fail. Last but not least, future researchers may want to continue investigating the field of neuroscience as there might be more valuable insights that can be applied and advance the business research fields. For example, the National Institutes of Health on September 16, 2013 released a fiftyeight page report that assess the state of neuroscience and offers a vision for the field's future (Marcus, 2013), a substantive step in developing United States President Barack Obama's \$100 million USD BRAIN Initiative (Brain Research Through Advancing Innovative Neurotechnologies) to unlock the mystery of the brain and understand the human mind. As Obama have rightly put it, "As humans, we can identify galaxies light years away, we can study particles smaller than an atom. But we still haven't unlocked the mystery of the three pounds of matter that sits between our ears." (Winerman, 2013). 


\section{REFERENCES}

Alarifi, S. \& Althonayan (2013), The Effects of Transformational Leadership on Followers' Creativity in Public Sector. 2nd International Conference on Management, Behavorial Sciences and Economics Issues.

Amabile, T (1982), “Children's artistic creativity: Detrimental effects of competition in a field setting".

Personality and Social Psychology Bulletin. Vol. 8. pp. 573-578.

Barinka, A (2014), IBM Wins Most U.S. Patents for 21st Year in a Row. http:// www.bloomberg.com/news/2014-01-14/ ibm-wins-most-u-s-patents-for-21 st-yearin-a- row.html (accessed January 25, 2014).

Batey, M., Chamorro-Premuzic, T., \& Furnham, A (2009), "Intelligence and personality as predictors of divergent thinking: The role of general, fluid and crystallized intelligence". Thinking Skills and Creativity, pp. 60-69.

Becker, W. Cropanzano, R. \& Sanfey, A (2011), “Organizational Neuroscience: Taking Organizational Theory beyond the Neural Black Box". Journal of Management. Vol. 37(4). pp. 933-961.

BTNRC (2013), What is Neuroscience? http:// www.btnrc.org/about-neuroscience/what-isneuroscience/ (accessed March 15, 2014).

Choi, H, \& Levine, J (2004), "Minority influence in work teams: The impact of newcomers". Journal of Experimental Social Psychology. Vol. 40. pp. 273-280.

Davis, G (1997), Identifying creative students and measuring creativity. In N. Colangelo \& G. A. Davis (Eds.), Handbook of gifted education (pp. 269-281): Pearson, Indiana: USA.
Dennis, A. \& Valachich, J (1993), "Computer brainstorming: More heads are better than one". Journal of Applied Psychology. Vol. 78, pp. 531-537.

Diehl, M. Stroebe, W(1987), "Productivity loss in brainstomirng groups. Toward the solution of a riddle". Journal of Personality and Social Psychology. Vol. 53 (3), pp. 497-509.

Dollinger, S. \& Clancy, S (1993), "Identity, self, and personality: II. Glimpses through the autographic eye". Journal of Personality and Social Psychology, Vol. 64(6), pp. 1064-71.

Dunnette, M. Campbell, J. \& Jaarstad, K (1963), "The effect of group participation on brain-storming effectiveness for two industrial samples". Journal of Applied Psychology, Vol. 47, pp. 30-37.

Feist, G (1998), "A meta-analysis of personality in scientific and artistic creativity". Personality and Social Psychology Review, Vol. 2, pp. 290-309.

Florida, J (2013), Managing Uncertainty and Succeeding in Today's Business Environment http://parallelliving. net/2013/09/17/managing-uncertaintyand-succeeding-in-todays-businessenvironment/ (accessed March 1, 2014).

Geroski, P. Machin, S. \&Van Reenen, J (1993), "The Profitability of Innovating Firms". RAND Journal of Economics. Vol 24, No. 2.

Gifted Education (2014), http://ststesting. com/2005giftttct.html (accessed May 21, 2014).

Gong, Y. Huang, J. \& Farh, J (2009), "Employee learning orientation, transformational leadership, and employee creativity: The mediating role of employee creative self-efficacy". Academy of Management Journal, Vol. 52, pp. 765-778. 
Goodreads Inc (2014), François La Rochefoucauld > Quotes > Quotable Quote. http://www.goodreads.com/ quotes/213766-the-only-thing-constantin-life-is-change (accessed March 1, 2014).

Howell, J. \& Higgins, C (1990), "Champions of Change: Identifying, Understanding, and Supporting Champions of Technological Innovations". Organizational Dynamics, Vol. 19, Issue 1, pp. 40.

IBM Sets U.S. Patent Record (2013). http://www-03.ibm.com/press/us/en/ pressrelease/40070.wss (accessed January 22, 2014).

Janis, I (1972), Victims of groupthink: Houghton Mifflin, Boston: USA.

Khan, R., Ur Rehman, A., \& Fatima, A (2009), "Transformational Leadership and Organizational Innovation: Moderated by Organizational Size". African Journal of Business Management, Vol. 3 (11), pp. 678- 684.

Kim, K (2006), "Can We Trust Creativity Test? A Review of the Torrance Tests of Creative Thinking (TTCT)". Creativity Research Journal, Vol. 18, No. 1, pp. 3-14.

King, L. Walker, L. \& Broyles, S (1996), "Creativity and the Five-Factor Model". Journal of Research in Personality, Vol. 30, Issue 2, pp 189-203.

Koetzier, W. \& Alon, A (2013), Why "Low Risk" Innovation Is Costly: Overcoming the Perils of Renovation and Invention. http://www.accenture. com/SiteCollectionDocuments/PDF/ Accenture- Why-Low-Risk-InnovationCostly.pdf (accessed January 5, 2014).

Marcus, G (2013), A Map for the Future of Neuroscience. http://amirttl.wordpress. com/2013/09/ (accessed on May 5, 2014).
Rock, D (2009), Managing with the Brain in Mind. http://www.strategy-business. com/article/09306?pg=all (accessed March 11, 2014).

Schutt, R (2012), Investigating the Social World: SAGE Publication: United Kingdom.

Taylor, D. Berry, P. \&Block, C (1958), "Does group participation when using brainstorming facilitate or inhibit creative thinking?". Administrative Science Quarterly, Vol. 6, pp. 22-47.

Thompson, M. Naccarato, M. Parker, K. \& Moskowitz, G (2001), The Personal Need for Structure (PNS) and Personal Fear of Invalidity (PFI) Scales: Historical Perspectives, Present Applications and Future Directions. In G. B. Moskowitz (Ed.), Cognitive Social Psychology: On the Tenure and Future of Social Cognition. (pp. 19-40): Lawrence Erlbaum Associates Publishers: USA.

Tjosvold, D. Tang, M. \& West, M (2004), Reflexivity for team innovation in China. Group \& Organization Management, Vol. 29, Pp. 540-559.

Webster, D. \& Kruglanski, A (1998), Cognitive and social consequences of the need for cognitive closure. European Review of Social Psychology, Vol. 8, pp. 133-173.

Winerman, L (2013), Decoding the Human Brain. http://www.apa.org/ monitor/2013/07-08/brain.aspx (accessed May 12,2014).

Wolfradt, U. \& Pretz, J (2001), Individual Differences in Creativity: Personality, Story Writing, and hobbies. European Journal of Personality, Vol. 15, Issue 4, pp. 297-310. 